\title{
A New Fairness-Oriented Packet Scheduling Scheme with Reduced Channel Feedback for OFDMA Packet Radio Systems
}

\author{
Stanislav NONCHEV, Mikko VALKAMA \\ Department of Communications Engineering, Tampere University of Technology, Tampere, Finland \\ Email: \{stanislav.nonchev, mikko.e.valkama\}@tut.fi \\ Received June 26, 2009; revised August 13, 2009; accepted September 26, 2009
}

\begin{abstract}
In this paper, we propose a flexible and fairness-oriented packet scheduling approach for 3GPP UTRAN long term evolution (LTE) type packet radio systems, building on the ordinary proportional fair (PF) scheduling principle and channel quality indicator (CQI) feedback. Special emphasis is also put on practical feedback reporting mechanisms, including the effects of mobile measurement and estimation errors, reporting delays, and CQI quantization and compression. The performance of the overall scheduling and feedback reporting process is investigated in details, in terms of cell throughput, coverage and resource allocation fairness, by using extensive quasi-static cellular system simulations in practical OFDMA system environment with frequency reuse of 1 . The performance simulations show that by using the proposed modified PF approach, significant coverage improvements in the order of $50 \%$ can be obtained at the expense of only $10-15 \%$ throughput loss, for all reduced feedback reporting schemes. This reflects highly improved fairness in the radio resource management (RRM) compared to other existing schedulers, without essentially compromising the cell capacity. Furthermore, we demonstrate the improved functionality increase in radio resource management for UE's utilizing multi-antenna diversity receivers.
\end{abstract}

Keywords: Radio Resource Management, Packet Scheduling, Proportional-Fair, Channel Quality Feedback, Throughput, Fairness

\section{Introduction}

Development of new radio interface technologies for beyond $3 \mathrm{G}$ cellular radio systems with support to high data rates, low latency and packet-optimised radio access has led to the use of OFDM/OFDMA. One good example of such developments is e.g. the UTRAN long term evolution (LTE), being currently standardized by $3 \mathrm{GPP}$ [1-3]. In general, performance improvements over the existing radio systems are basically obtained through proper deployment of fast link adaptation and new packet scheduling algorithms, exploiting the available multi-user diversity in both time and frequency domains [4-6]. On the other hand, achieving such performance improvements typically requires relatively accurate channel state feedback in terms of CQI reports from mobile stations (MS) to the base station (BS) [6-12]. As a practical example, each mobile station can measure the effective signal-to-interference-plus-noise-ratio (SINR), per active subcarrier or block of subcarriers, and send back the obtained channel state to the base station for downlink radio resource management. This, in turn, can easily lead to considerable control signalling overhead if not designed and implemented properly. Thus in general, the amount of the feedback information needs to be limited and is also subject to different errors and delays, affecting the overall system-level performance. Another important aspect in scheduling and resource allocation process is fairness, implying that also users with less favourable channel conditions should anyway be given some reasonable access to the radio spectrum [4-6,1318]. This is especially important in serving users at, e.g., cell edges in cellular networks.

In this paper, we address the packet scheduling and channel state reporting tasks in OFDMA-based cellular packet radio systems. Stemming from ordinary proportional fair (PF) scheduling principle, a modified PF scheduler is first proposed having great flexibility to tune the exact scheduling characteristics in terms of capacity, coverage and fairness. More specifically, the proposed 
scheduler can offer greatly improved fairness among the users in a cell, measured in terms of coverage and other established fairness measures, like Jain's index [19], without essentially compromising the overall cell capacity. This is verified using extensive quasi-static cellular system simulations, conforming to the current LTE downlink specifications [1-3]. In the performance studies, different realistic CQI reporting schemes are also addressed and incorporated in the system simulations.

In general, the research on novel packet scheduling algorithms and channel state reporting schemes has been very active in the recent years, see e.g. $[8,10,11,13-18]$ and the references therein. Using [13-17] as starting points for LTE type packet radio systems, it has been reported that frequency domain packet scheduling (FDPS) algorithms are always a compromise between the overall cell throughput and resource fairness among users. Here we propose a modified proportional fair algorithm, which in general offers an attractive balance between cell throughput, coverage and user fairness. Compared to plain frequency domain scheduling, we extend the studies by deploying both time domain and frequency domain scheduling steps, together with proper metrics, that as a whole can more efficiently utilise the provided yet limited feedback information from all the user equipments (UEs). Furthermore, we apply different realistic CQI reporting schemes to thoroughly investigate the limits of achieved performance gains from enhanced scheduling. The cellular system model used for the performance evaluations is fully conforming to the $3 \mathrm{GPP}$ evaluation criteria [1-3]. The overall outcomes are measured in terms of average cell throughput, coverage and fairness index.

The rest of the paper is organised as follows: Section 2 reviews the reference proportional fair scheduler and proposes then a modified PF scheduling scheme. Section 3 , in turn, addresses different feedback reporting schemes in the scheduling context. Section 4 presents then the overall system model and simulation assumptions, and the simulation results and analysis are presented in Section 5. Finally, the conclusions are drawn in Section 6.

\section{Scheduling Process}

\subsection{General Scheduling and Link Adaptation Principles}

In general, the task of a packet scheduler (PS) is to select the most suitable users to access the available radio spectrum at any given time window, in order to optimize the system performance in terms of 1) throughput, 2) resource fairness, and/or 3) delay [4-6]. Joint optimization of all the above features is generally known very difficult. In fast packet scheduling, new scheduling decisions are basically taken in each transmission time interval (TTI), which in LTE is $1 \mathrm{~ms}$.

To efficiently utilize the limited radio resources, the scheduler should consider the current state of the channel when selecting the user to be scheduled, by utilizing e.g. the ACK/NACK signalling information and CQI reports $[4-6,8,10,11,14]$. Depending on the selected CQI reporting scheme, the accuracy and resolution of the channel quality information can easily differ considerably. In OFDMA based radio systems, like LTE, the CQI information is not necessarily available for all the individual subcarriers but more likely for certain groups of subcarriers only [12,20-22]. In general, the channel state information is also used by link adaptation (LA) mechanisms to select proper modulation and coding scheme (MCS) for each scheduled mobile, and thereon to ensure that the individual link qualities conform to the corresponding target settings. This is typically measured in terms of block error rate (BLER) for the first transmission. Hybrid ARQ (HARQ) mechanisms are then commonly used to provide the necessary buffer information and transmission format for pending retransmissions $[4-6,16]$. A principal block-diagram of the overall RRM flow is given in Figure 1.

As a practical example of the available spectral resources, in the $10 \mathrm{MHz}$ system bandwidth case of LTE [1-3], there are 50 physical resource blocks (PRB's or sub-bands), each consisting of 12 sub-carriers with subcarrier spacing of $15 \mathrm{kHz}$. This sets the basic resolution in frequency domain (FD) UE multiplexing (scheduling), i.e., the allocated individual UE bandwidths are multiples of the PRB bandwidth.

\subsection{Ordinary Proportional Fair (PF) Scheduler}

The well-known proportional fair scheduler $[13,16]$ works in two steps: 1) time domain (TD) PF step and 2) frequency domain (FD) PF step. Such simplified sche-

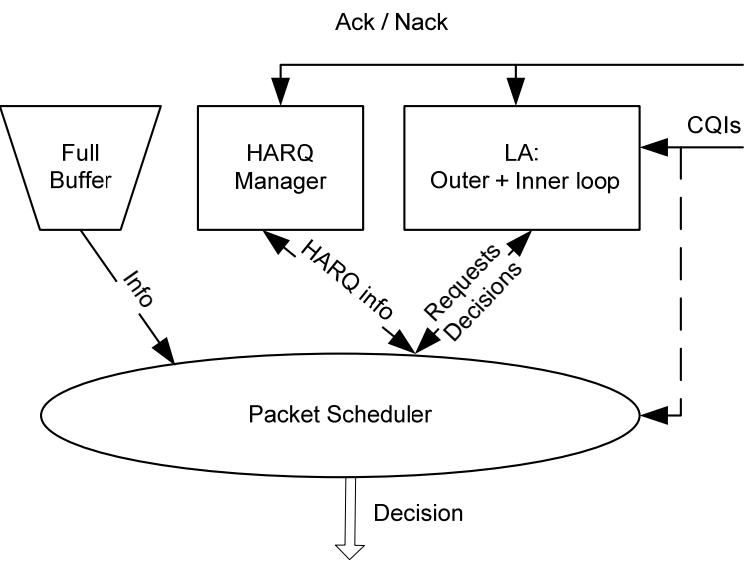

Figure 1. Principal RRM block diagram. 
duling principle is beneficial from the complexity point of view, since the FD step considers a reduced number of UEs for frequency multiplexing in each TTI [17]. Thus in the first part, inside each TTI $n$, all the UE's are ranked according to the following priority metric

$$
\gamma_{i}^{t d}(n)=\frac{R_{i}(n)}{T_{i}(n)}
$$

In above, the UE index $i=1,2, \ldots, I_{\mathrm{TOT}}, R_{i}(n)$ denotes the estimated throughput to the UE $i$ over the full bandwidth (provided by link adaptation unit) $[13,16]$, and $T_{i}(n)$ in turn is the corresponding average delivered throughput to the UE $i$ during the recent past and can be obtained, e.g., recursively by

$$
T_{i}(n)=\left(1-\frac{1}{t_{c}}\right) T_{i}(n-1)+\frac{1}{t_{c}} R_{i}^{\prime}(n-1)
$$

In (2), $t_{c}$ controls the averaging window length over which the average delivered throughput is calculated and $R_{i}^{\prime}(n-1)$ denotes the actually realized throughput to the UE $i$ at the previous TTI.

In the next step, out of this ranked list of UE's, the first $I_{\mathrm{BUFF}}\left(<I_{\mathrm{TOT}}\right)$ UE's with highest priority metric are picked to the actual frequency domain multiplexing or scheduling stage. In the following, this subset is called scheduling candidate set (SCS), and is denoted by $\Omega(n)$. Then, for each physical resource block $k=1,2, \ldots, K_{\mathrm{TOT}}$, and for each $i$ belonging to the SCS, the following final scheduling metric of the form

$$
\gamma_{i, k}^{f d}(n)=\frac{R_{i, k}(n)}{T_{i}(n)}
$$

is evaluated where now $R_{i, k}(n)$ denotes the estimated throughput to the UE $i$ for the $k$-th PRB (provided by LA unit again), and $T_{i}(n)$ is again the corresponding average throughput delivered to the UE $i$ during the recent past given in (2). Finally, the access to each PRB resource is granted for the particular user with the highest metric for the corresponding PRB.

\subsection{Proposed Modified PF (MPF) Scheduler}

In order to obtain a scheduler with yet increased fairness in the resource allocation, we proceed as follows. First the time domain priority metric is modified as

$$
\bar{\gamma}_{i}^{t d}(n)=C Q I_{i}(n)\left(\frac{T_{i}(n)}{T_{t o t}(n)}\right)^{-1}
$$

where $C Q I_{i}(n)$ denotes the full bandwidth channel quality report for UE $i$ at TTI $n$ and $T_{i}(n)$ is as defined in (2). $T_{t o t}(n)$, in turn, denotes the averaged throughput over the past and over the scheduled users and can be calculated by

$$
\begin{aligned}
T_{\text {tot }}(n)= & \left(1-\frac{1}{t_{c}}\right) T_{\text {tot }}(n-1) \\
& +\frac{1}{t_{c}} \frac{1}{I_{B U F F}} \sum_{i \in \Omega(n-1)} R_{i}^{\prime}(n-1)
\end{aligned}
$$

In (5), $R_{i}^{\prime}(n-1)$ denotes the actual delivered throughput for UE $i$ at the previous TTI.

Similar to the ordinary PF scheduler described in Subsection 2.2, this modified metric in (4) is used to rank the UE's inside each TTI, and the $I_{\mathrm{BUFF}}\left(<I_{\mathrm{TOT}}\right)$ UE's with highest priority metric form a SCS. $\Omega(n)$ for the actual frequency domain resource allocation. Since estimated throughput in the link adaptation stage is based on reported CQI values, we assume that the substitution in (4) has the same weight in priority calculation. For mapping the users of the SCS into PRB's, the following modified frequency domain metric is then proposed:

$$
\bar{\gamma}_{i, k}^{f d}(n)=\left(\frac{C Q I_{i, k}(n)}{C Q I_{i}^{a v g}(n)}\right)^{S_{1}}\left(\frac{T_{i}(n)}{T_{t o t}(n)}\right)^{-S_{2}}
$$

Here $s_{1}$ and $s_{2}$ are adjustable parameters, and $C Q I_{i, k}(n)$ is the channel quality report of user $i$ for sub-band $k$ at TTI $n$ while $C Q I_{i}^{a v g}(n)$ is the corresponding average CQI over the past and over the sub-bands, and can be calculated using

$$
\begin{aligned}
C Q I_{i}^{a v g}(n) & =\left(1-\frac{1}{t_{c}}\right) C Q I_{i}^{a v g}(n-1) \\
+ & \frac{1}{t_{c}} \frac{1}{K_{T O T}} \sum_{k=1}^{K_{T O T}} C Q I_{i, k}(n)
\end{aligned}
$$

The access to each PRB resource is then granted for the particular user with the highest metric in (6) for the corresponding PRB.

Considering the re-transmissions, re-transmitting users are simply considered as additional users in the time domain scheduling part (step 1), and if qualified to the frequency domain SCS, the re-transmission users are given an additional priority to reserve exactly the same subbands used for the corresponding original transmissions. Even though this does not take the exact sub-band condition into account at re-transmission stage, the practical implementation is simplified, in terms of control signalling, and re-transmissions anyway always benefit from the HARQ combining gain [6].

Intuitively, the proposed scheduling metrics in (4) and (6) are composed of two elements, affecting the overall scheduling decisions. The first dimension measures the relative instantaneous quality of the individual user's radio channels against their own average channel qualities while the second dimension is related to measuring the achievable throughput of individual UE's against the corresponding average throughput of scheduled users. Consequently, by understanding the power coefficients $s_{1}$ 
and $s_{2}$ as additional adjustable parameters, the exact scheduler statistics can be tuned and controlled to obtain a desired balance between the throughput and fairness. This will be demonstrated in Section 5 .

\section{Feedback Reporting Process}

The overall reporting process between UE's and BS is illustrated in Figure 2. Within each time window of length $t_{r}$, each mobile sends channel quality indicator (CQI) reports to BS, formatted and possibly compressed, with a reporting delay of $t_{d}$ seconds $[6,8,10,11]$. Each report is naturally subject to errors due to imperfect decoding of the received signal. In general, the CQI reporting frequency-resolution has a direct impact on the achievable multi-user frequency diversity and thereon to the overall system performance and the efficiency of radio resource management (RRM), as described in general e.g. in [11]. In our studies here, the starting point (reference case) is that the CQI reports are quantized SINR measurements across the entire bandwidth (wideband CQI reporting), to take advantage of the time and frequency variations of the radio channels for the different users. Then also alternative reduced feedback schemes are described and evaluated, as discussed below.

\subsection{Full CQI Reporting}

In a general OFDMA radio system, the overall system bandwidth is assumed to be divided into $v$ CQI measurement blocks. Then quantizing the CQI values to $q$ bits, the overall full CQI report size is

$$
S_{\text {full }}=q \times v
$$

bits which is reported by every UE for each TTI $[1-3,11]$. In case of LTE, with $10 \mathrm{MHz}$ system bandwidth and grouping 2 physical resource blocks into 1 measurement

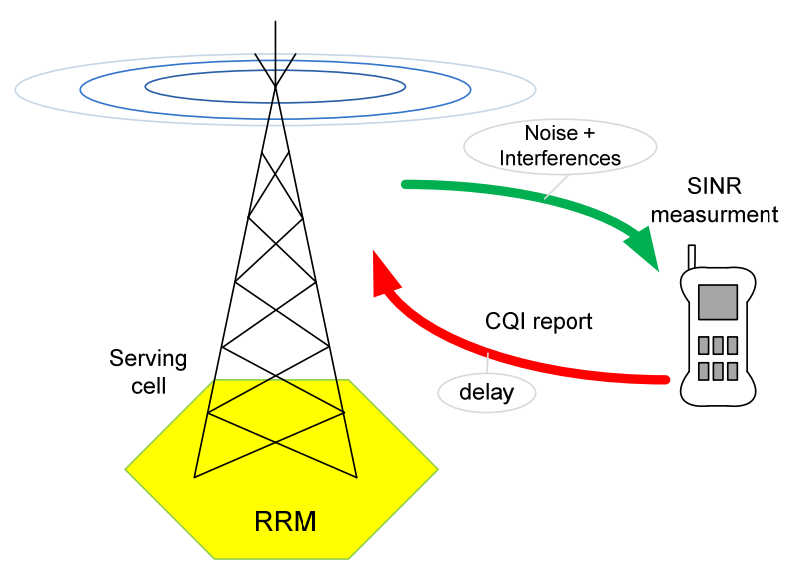

Figure 2. Reporting mechanism between UE and BS. block, it follows that $v=25$. Assuming further that quantization is carried with $q=5$ bits, then each UE is send ing $25 \times 5=125$ bits for every $1 \mathrm{~ms}$ (TTI length).

\subsection{Best-m CQI Reporting}

One simple approach to reduce the reporting and feedback signalling is obtained as follows. The method is based on selecting only $m<v$ different CQI measurements and reporting them together with their frequency positions to the serving cell $[8,11]$. We assume here that the evaluation criteria for choosing those $m$ sub-bands for reporting is based on the highest SINR values (hence the name best-m). The resulting report size in bits is then given by

$$
S_{\text {best-m }}=q \times m+\left\lceil\log _{2}\left(\frac{v !}{m !(v-m) !}\right)\right\rceil
$$

As an example, with $v=25, q=5$ bits and $m=10$, it follows that $S_{\text {best-m }}=72$ bits, while $S_{\text {full }}=125$ bits. Furthermore, on the scheduler side, we assume that the PRBs which are not reported by the UE are allocated a $\mathrm{CQI}$ value equal to the lowest reported one.

\subsection{Threshold Based CQI Reporting}

This reporting scheme is a further simplification and relies on providing information on only the average CQI value above certain threshold together with the corresponding location (sub-band index) information. First the highest CQI value is identified within the full bandwidth, which sets an upper bound of the used threshold window. All CQI values within the threshold window are then averaged and only this information is sent to the BS together with the corresponding sub-band indexes. On the scheduler side, the missing CQI values can then be treated, e.g., as the reported averaged CQI value minus a given $\mathrm{dB}$ offset (e.g. $5 \mathrm{~dB}$, the exact number is again a design parameter). The number of bits needed for reporting is therefore only

$$
S_{\text {threshold }}=q+v
$$

As an example, with $v=25$ and $q=5$ bits (as above), it follows that $S_{\text {threshold }}=30$ bits, while $S_{\text {best }-m}=72$ bits and $S_{\text {full }}=125$ bits. The threshold-based scheme is illustrated graphically in Figure 3 [10].

\section{System Simulation Model and Assumptions}

In order to evaluate the system-level performance of the proposed scheduling scheme in a practical OFDMA-based cellular system context, a comprehensive quasi-static system simulator for LTE downlink has been developed, 


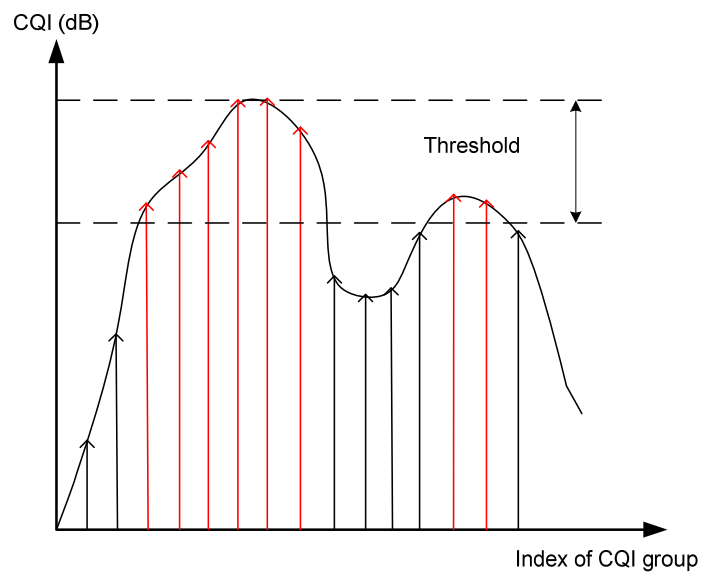

Figure 3. Basic principle of threshold-based CQI reporting.

conforming to the specifications in [1-3]. In the overall simulation flow, mobile stations are first randomly dropped or positioned over each sector and cell. Then based on the individual distances between the mobiles and the serving base station, the path losses for individual links are directly determined, while the actual fading characteristics of the radio channels depend on the assumed mobility and power delay profile. In updating the fading statistics, the time resolution in our simulator is set to one TTI $(1 \mathrm{~ms})$. In general, a standard hexagonal cellular layout is utilized with altogether 19 cell sites each having 3 sectors. In the performance evaluations, statistics are collected only from the central cell site while the others simply act as sources of inter-cell interference.

As a practical example case, the $10 \mathrm{MHz}$ LTE system bandwidth mode [1-3] is assumed. The main simulation parameters and assumptions are generally summarized in Table 1 for the so-called Macro cell case 1, following again the LTE working assumptions. As illustrated in Figure 1, the RRM functionalities are controlled by the packet scheduler and also link adaptation and HARQ mechanisms are modelled and implemented, as described in Table 1. As a practical example, the maximum number of simultaneously multiplexed users $\left(I_{\mathrm{BUFF}}\right)$ is set to 10 here. In general, we assume that the BS transmission power is equally distributed among all PRB's. In the basic simulations, 20 UE's are uniformly dropped within each sector and experience inter-cell interferences from the surrounding cells, in addition to path loss and fading. The UE velocity equals $3 \mathrm{~km} / \mathrm{h}$, and the typical urban (TU) channel model standardized by ITU is assumed in modelling the power-delay spread of the radio channels. Infinite buffer traffic model is applied in the simulations, i.e. every user has data to transmit (when scheduled) for the entire duration of a simulation cycle. The length of a single simulation run is set to 5 seconds which is then repeated for 10 times to collect reliable statistics.

In general, every UE has an individual HARQ entry,
Table 1. Basic simulation parameters.

\begin{tabular}{|c|c|}
\hline Parameter & Assumption \\
\hline Inter-site distance & $\begin{array}{l}\text { Hexagonal grid, } 19 \text { cell } \\
\text { sites, 3 sectors per site } \\
500 \mathrm{~m}\end{array}$ \\
\hline $\begin{array}{l}\text { Carrier Frequency / Bandwidth } \\
\text { Number of active sub-carriers } \\
\text { Sub-carrier spacing } \\
\text { Sub-frame duration } \\
\text { Channel estimation }\end{array}$ & $\begin{array}{l}2000 \mathrm{MHz} / 10 \mathrm{MHz} \\
600 \\
15 \mathrm{kHz} \\
0.5 \mathrm{~ms} \\
\text { Ideal }\end{array}$ \\
\hline PDP & ITU Typical Urban 20 paths \\
\hline $\begin{array}{l}\text { Minimum distance between UE } \\
\text { and cell } \\
\text { Average number of UE's per sector }\end{array}$ & $\begin{array}{l}>=35 \text { meters } \\
20\end{array}$ \\
\hline $\begin{array}{l}\text { Max. number of frequency multi- } \\
\left.\text { plexed UEs ( } \mathrm{I}_{\mathrm{BIFF}}\right) \\
\text { UE receiver type }\end{array}$ & 2-Rx MRC, 2-Rx IRC \\
\hline Shadowing standard deviation & $8 \mathrm{~dB}$ \\
\hline UE speed & $3 \mathrm{~km} / \mathrm{h}$ \\
\hline Total BS TX power $\left(\mathrm{P}_{\text {total }}\right)$ & $46 \mathrm{dBm}$ \\
\hline Traffic model & Full Buffer \\
\hline Fast Fading Model & Jakes Spectrum \\
\hline CQI reporting schemes & $\begin{array}{l}\text { Full CQI } \\
\text { Best-m (with } \mathrm{m}=10 \text { ) } \\
\text { Threshold based (with } 5 \mathrm{~dB} \\
\text { threshold) }\end{array}$ \\
\hline CQI log-normal error std. & $1 \mathrm{~dB}$ \\
\hline CQI reporting time & 5 TTI \\
\hline $\begin{array}{l}\text { CQI delay } \\
\text { CQI quantization } \\
\text { CQI std error } \\
\text { MCS rates }\end{array}$ & $\begin{array}{l}2 \text { TTIs } \\
1 \mathrm{~dB} \\
1 \mathrm{~dB} \\
\text { QPSK }(1 / 3,1 / 2,2 / 3) \text {, } \\
\text { 16QAM }(1 / 2,2 / 3,4 / 5) \text {, } \\
\text { 64QAM }(1 / 2,2 / 3,4 / 5)\end{array}$ \\
\hline ACK/NACK delay & $2 \mathrm{~ms}$ \\
\hline Number of SAW channels & 6 \\
\hline Maximum number of retransmisions & 3 \\
\hline HARQ model & Ideal chase combining (CC) \\
\hline $1^{\text {st }}$ transmission BLER target & $20 \%$ \\
\hline Scheduler forgetting factor & 0.002 \\
\hline Simulation duration (one drop) & $\begin{array}{l}\text { Ordinary PF (for reference) } \\
\text { Modified PF } \quad \text { (proposed } \\
5 \text { seconds }\end{array}$ \\
\hline Number of drops & 10 \\
\hline
\end{tabular}

which operates the physical layer re-transmission functionalities. It is based on the stop-and-wait (SAW) protocol and for simplicity, the number of entries per UE is fixed to six. HARQ retransmissions are always transmitted with the same MCS and on the same PRB's (if scheduled in TD step) as the first transmissions. The supported modulation schemes are QPSK, 16QAM and 64QAM with variable rates for the encoder as shown in Table 1.

Link adaptation handles the received UE reports con- 
taining the channel quality information for the whole or sub-set of PRB's as described in Section 3. The implemented link adaptation mechanism consists of two separate elements - the inner loop (ILLA) and outer loop (OLLA) LA's - and are used for removing CQI imperfections and estimating supported data rates and MCS. As a practical example, it is assumed that the CQI report errors are log-normal distributed with $1 \mathrm{~dB}$ standard deviation.

The actual effective SINR calculations rely on estimated subcarrier-wise channel gains (obtained using reference symbols in practice) and depend in general also on the assumed receiver topology. Here we assume the single-input-multiple-output (SIMO) diversity reception case, i.e. a single BS transmit antenna and multiple UE receiver antennas. Considering now an individual UE $i$, the SINR per active sub-carrier $c$ at TTI $n$, denoted here by $\xi_{i, c}(n)$, is calculated according to

$$
\xi_{i, c}=\frac{\left|\mathbf{w}_{i, c}^{H} \mathbf{h}_{i, c}\right|^{2} \sigma_{s i g, i}^{2}}{\mathbf{w}_{i, c}^{H} \sum_{n o i s e} \mathbf{w}_{i, c}+\mathbf{w}_{i, c}^{H} \sum_{i n t, i} \mathbf{w}_{i, c}}
$$

where the time index $n$ is dropped for notational simplicity. Here $\mathrm{h}_{i c}$ is an $N_{R X} \mathrm{x} 1$ vector of the user $i$ complex channel gains at subcarrier $c$ from BS to $N_{R X}$ receiver antennas and $\mathrm{w}_{i c}$ is the corresponding $N_{R X} \mathrm{X} 1$ spatial filter used to combine the signals of different receiver antennas (more details below). $s_{\text {sig, } i}^{2}$, in turn, denotes the received nominal signal power per antenna while $\boldsymbol{\Sigma}_{\text {noise }}$ and $\boldsymbol{\Sigma}_{i n t, i}$ are the covariance matrices of the received (spatial) noise and interference vectors. The superscript $(.)^{H}$ denotes conjugate transpose. The noise covariance is assumed diagonal ( $\left.\boldsymbol{\Sigma}_{\text {noise }}=s_{\text {noise }}^{2} \mathbf{I}\right)$ and independent of the user index $i$. The interference modeling, on the other hand, takes into account the interference from neighboring cells. Assuming a total of $L_{\text {int }}$ interference sources, with corresponding path gain vectors $\mathbf{g}_{l, i, c}$, the overall interference covariance at receiving UE $i$ is given by

$$
\mathrm{S}_{i n t, i}=\sum_{l=1}^{L_{\text {int }}} \sigma_{i n t, l, i}^{2} \mathbf{g}_{l, i, c} \mathbf{g}_{l, i, c}^{H}
$$

where $s_{\text {int }, l, i}^{2}$, denotes the received nominal interferer power per antenna and per interference source $(l)$.

Concerning the actual UE receiver topologies (spatial filters), both maximum ratio combining (MRC) and interference rejection combining (IRC) receivers are deployed in the simulations. These are given by (see, e.g., [6] and the references therein)

$$
\mathbf{w}_{i, c}^{M R C}=\frac{\mathbf{h}_{i, c}}{\left\|\mathbf{h}_{i, c}\right\|^{2}}
$$

and

$$
\mathbf{w}_{i, c}^{I R C}=\frac{\sum_{t o t, i}^{-1} \mathbf{h}_{i, c}}{\mathbf{h}_{i, c}^{H} \sum_{t o t, i}^{-1} \mathbf{h}_{i, c}}
$$

where $\boldsymbol{\Sigma}_{t o t, i}$ denotes the total noise plus interference covariance, i.e., $\boldsymbol{\Sigma}_{t o t, i}=s_{\text {noise }}^{2} \mathbf{I}+\boldsymbol{\Sigma}_{\text {int }, i}$.

Using the above modeling and the selected UE receiver type, the effective SINR values are then calculated through exponential effective SINR mapping (EESM), as described in [1-3], for link-to-system level mapping purposes.

\section{Results}

In this section, we present the system-level performance results obtained using the previously described quasistatic radio system simulator. Both ordinary $\mathrm{PF}$ and modified (proposed) PF packet schedulers are used, together with the three different CQI reporting schemes. The system-level performance is generally measured and evaluated in terms of:

- Throughput statistics - the cumulative distribution function (CDF) of the total number of successfully delivered bits per time unit. Measured at both individual UE level as well as overall cell level.

- Coverage - the experienced data rate per UE at the $95 \%$ coverage probability (5\% UE throughput CDF level).

- Jain's fairness index [19].

In addition to Jain's index, also the coverage and slope of the throughput CDF reflect the fairness of the scheduling algorithms.

With the proposed modified PF scheduler, different example values for the power coefficients $s_{1}$ and $s_{2}$ are used as shown in Table 2. To focus mostly on the role of the channel quality reporting, $s_{2}$ is fixed here to 1 and the effects of using different values for $s_{1}$ are then demonstrated. This way the impact of the different CQI reporting schemes is seen more clearly. For the cases of Best- $m$ and Threshold based CQI reporting schemes, we fix the value of $m$ equal to 10 and threshold to $5 \mathrm{~dB}$, respectively. Similar example values have also been used by other authors in the literature earlier, see e.g. [11]. Complete performance statistics are gathered for both dual antenna MRC and dual antenna IRC UE receiver cases.

Table 2. Different power coefficient combinations used to evaluate the performance of the proposed scheduler.

\begin{tabular}{cccccccc}
\hline Coefficient & \multicolumn{7}{c}{ Value } \\
\hline $\mathrm{s}_{1}$ & 1 & 2 & 4 & 6 & 8 & 10 & 20 \\
$\mathrm{~s}_{2}$ & 1 & 1 & 1 & 1 & 1 & 1 & 1 \\
\hline
\end{tabular}




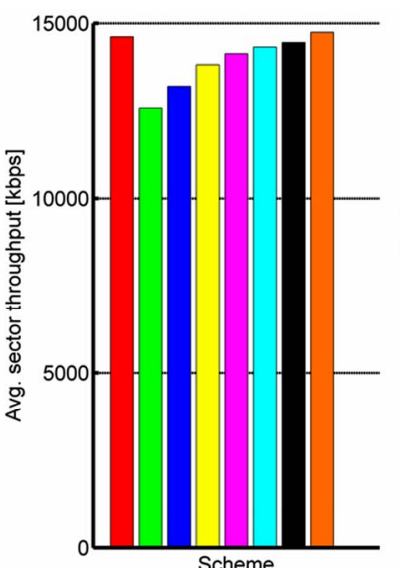

(a)

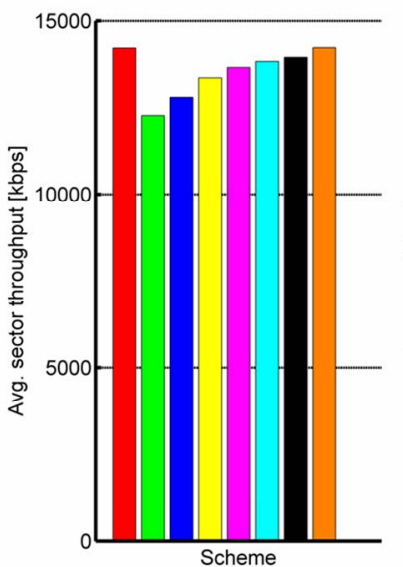

(c)

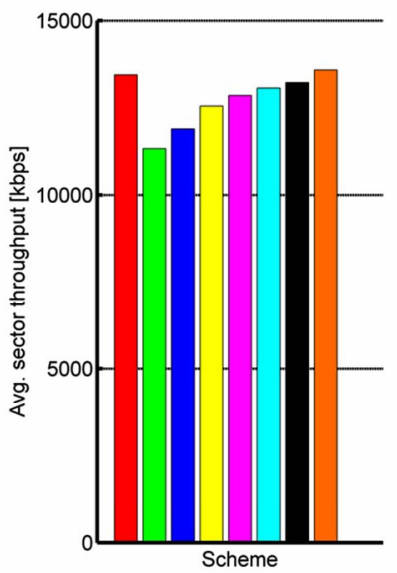

(e)

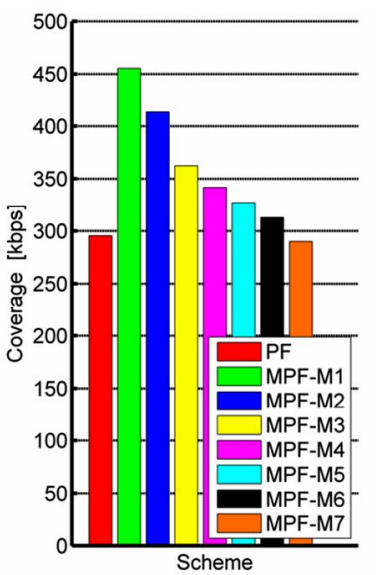

(b)

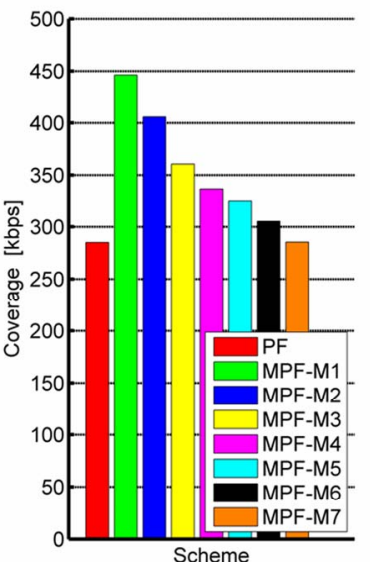

(d)

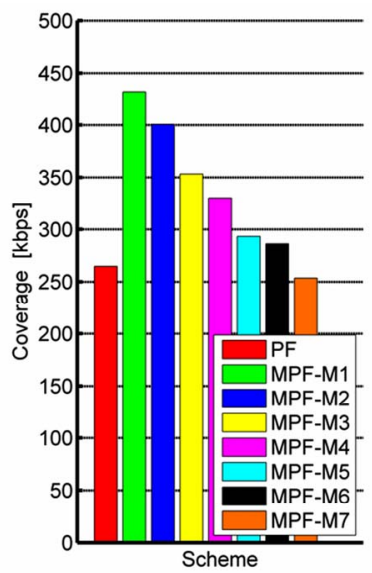

(f)

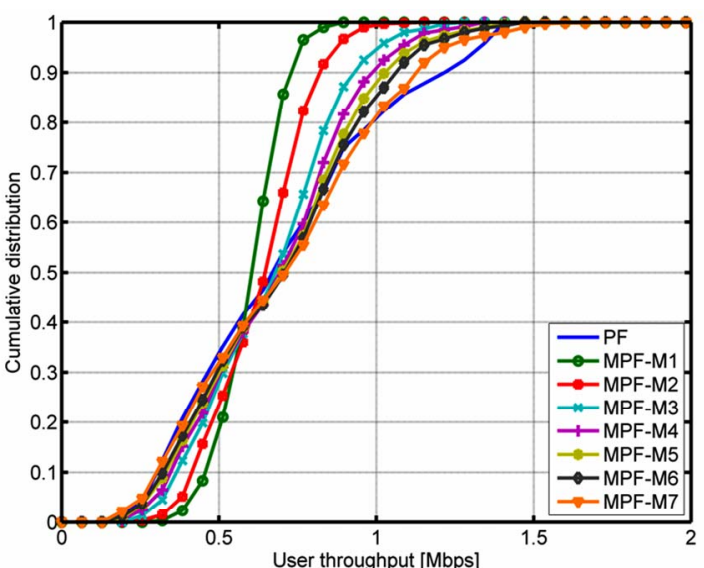

(a)

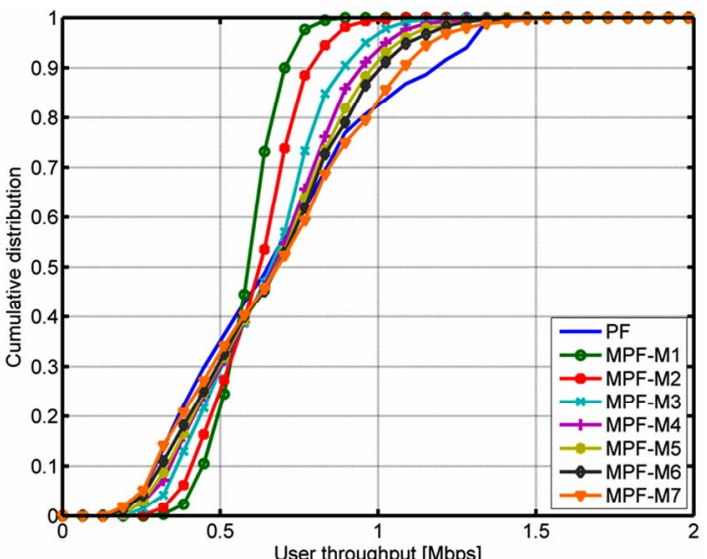

(b)

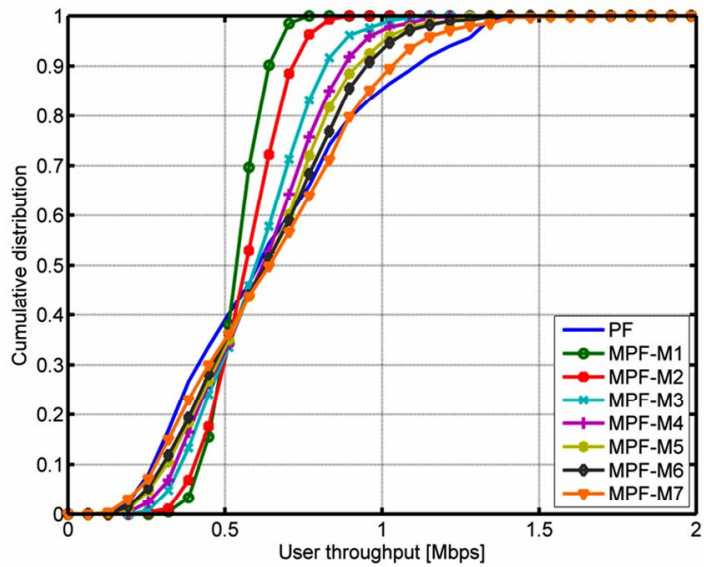

(c)

Figure 4. Left column: Average sector throughput and coverage for different scheduling schemes and assuming dual-antenna MRC UE receiver type with full CQI feedback (a, b), Best -m CQI feedback (c, d) and Threshold based CQI feedback (e, f). M1-M7 refer to the modified PF scheduler with power coefficient values as given in Table 2 (M1: $s_{1}=1$, $s_{2}=1$, etc.). Right column: CDF's of individual UE throughputs for different scheduling schemes and assuming dual-antenna MRC UE receiver type with full CQI feedback (a), Best -m CQI feedback (b) and Threshold based CQI feedback (c).

\subsection{Dual Antenna MRC UE Receiver Case}

Figure 4 (left column) illustrates the average sector throughput and coverage for the different schedulers, assuming dual antenna maximum ratio combining (MRC) UE receiver type. The power coefficient values from Table 2 are presented as index M, where M1 represents the first couple $\left(s_{1}=1, s_{2}=1\right)$, etc, for the metric calcula- 
tion of the modified PF scheduler. The used reference scheduler is the ordinary proportional fair approach. In the first coefficient case (M1), in combination with full CQI reporting scheme, we achieve coverage gain in the order of $50 \%$ at the expense of only $15 \%$ throughput loss as shown

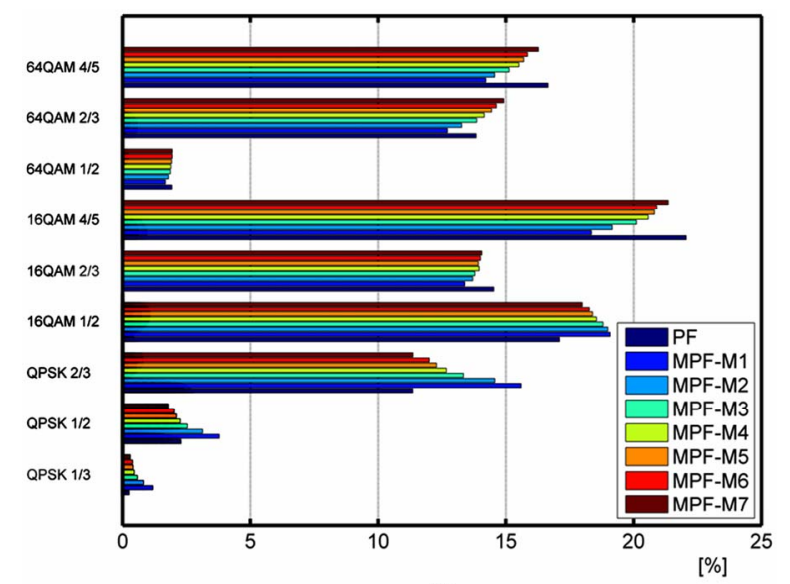

(a)

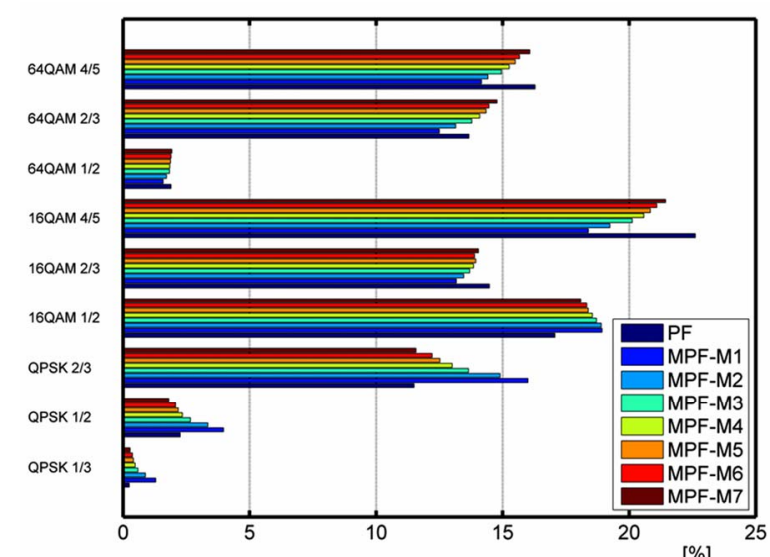

(b)

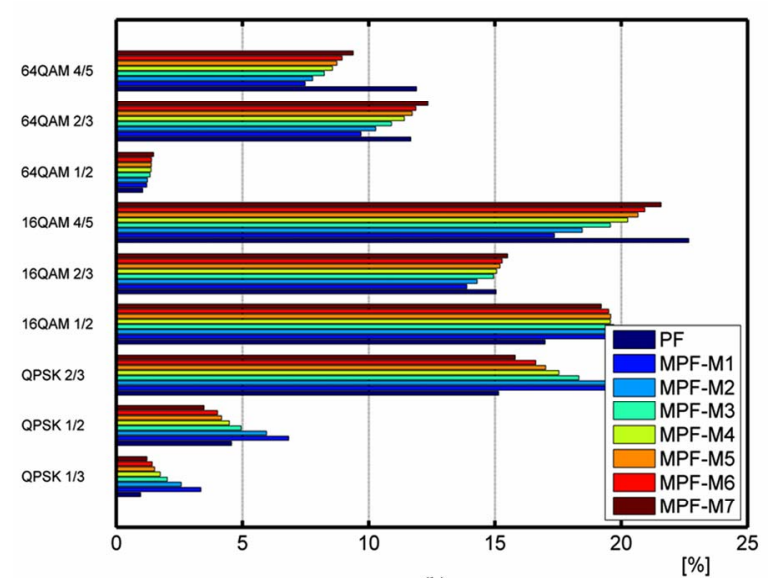

(b) in Figure 4 (a) and (b). This sets the basic reference for comparisons in the other cases. In the case of best-m and threshold based reporting schemes presented in and (d), and Figure 4 (e) and (f), we have coverage increases by $57 \%$ and $63 \%$ with throughput losses of $16 \%$ and $19 \%$, correspondingly.

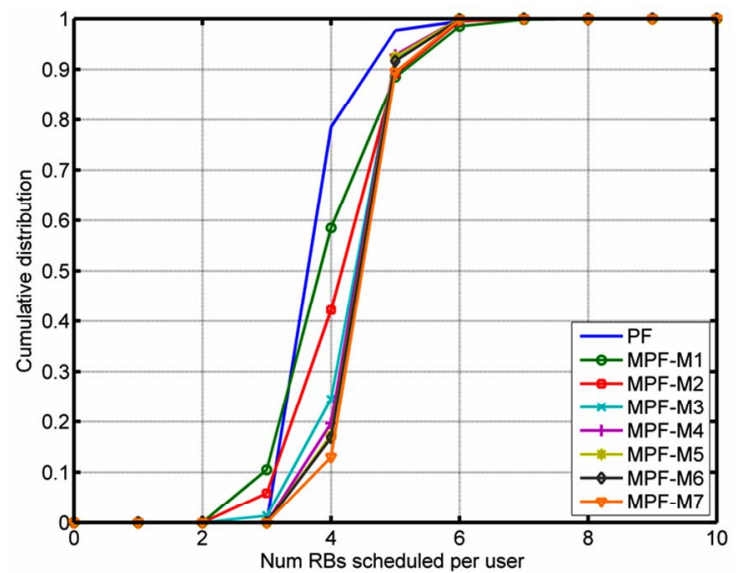

(a)

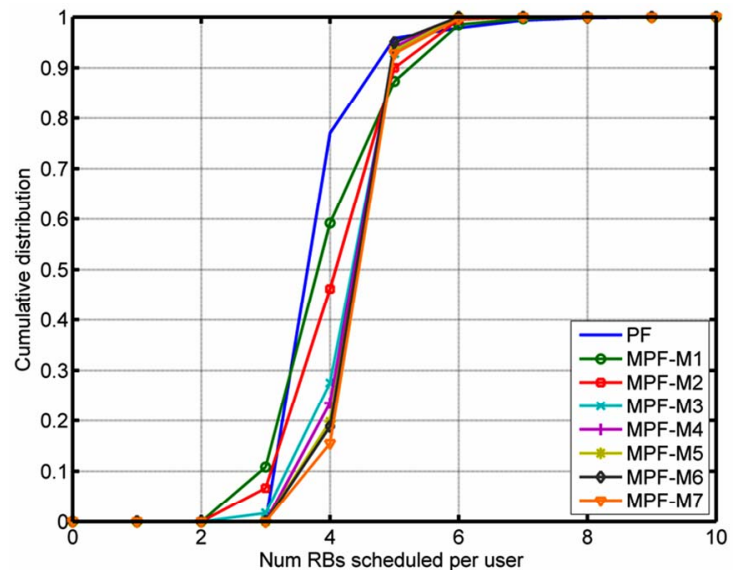

(b)

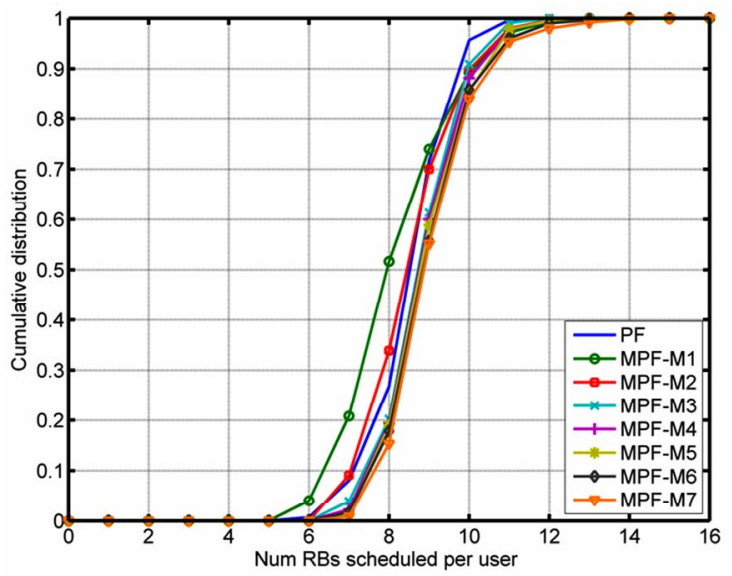

(c)

Figure 5. Left column: MCS distributions [\%] for different scheduling principles with (a) Full CQI reporting, (b) Best-m CQI reporting, and (c) Threshold based CQI reporting assuming dual-antenna MRC UE receiver. Right column: CDF's of scheduled PRB's per user for different schedulers with (a) Full CQI reporting, (b) Best-m CQI reporting, and (c) Threshold based CQI reporting assuming dual-antenna MRC UE receiver. 
Continuing on the evaluation of relative system performance using the modified PF scheduler, we clearly see a trade-off between average cell throughput and coverage for different power coefficient cases. The remaining power coefficient values shown in Table 2 are used for tuning the overall system behaviour together with the choice of the CQI reporting scheme. In the case of full CQI feedback and coefficient $s_{1}$ varying between 2 and 10 (M2-M6) the cell throughput loss is decreased to around $1 \%$, while the coverage gain is reduced to around $6 \%$. Similar behaviour is observed for the other feedback reporting schemes as well. The exact percentage values for the coverage gains and throughput losses are stated in Table 3 in the end.

Further illustrations on the obtainable system performance are presented in Figure 4 (right column) in terms of the statistics of individual UE data rates for the applied simulation scenarios. The slope of the CDF reflects generally the fairness of the algorithms. Therefore we aim to achieve steeper slope corresponding to algorithm fairness. This type of slope change behavior can clearly be established for each simulation scenario. Clearly, at 5\% (coverage) point of the CDF curves, corresponding to users typically situated at the cell edges, we observe significant data rate increases indicated by shift to the right for all CQI feedback schemes when the coefficient $s_{1}$ is changed in the proposed metric. This indicates improved overall cell coverage at the expense of slight total throughput loss.
Figure 5 (left column) shows the modulation and coding scheme (MCS) distributions for different schedulers and with applied feedback reporting schemes, still assuming the case of 2 antenna MRC UE receiver type. The negligible decrease in higher order modulation usage (less than 3\%) leads to the increase in the lower (more robust) ones for improving the cell coverage. In all the simulated cases, the MCS distribution behaviour has a relatively similar trend following the choice of the power coefficients in the proposed packet scheduling. In general, the use of higher-order modulations is affected mostly in the most coarse CQI feedback (threshold based) case while the other two reporting schemes behave fairly similarly.

Similarly, Figure 5 (right column) illustrates the CDF's of scheduled PRB's per UE for the different scheduler scenarios and reporting schemes. Clearly, the modified PF provides better resource allocation in the full and best-m feedback cases. Considering the 50\% probability point for the resource allocation, and taking the case of M1, we have about 5\% gain, while in case of M2 the gain is raised to $15 \%$ compared to ordinary PF. The average obtained improvement for the rest of the cases is about $33 \%$. In the case of threshold-based feedback, the resource allocation is not as efficient, and even a small reduction in the RB allocation is observed with small power coefficients, compared to the reference PF scheduler. Starting from M3, the improvement is anyway noticeable and the achieved gain is about $20 \%$.

Table 3. Obtained performance statistics compared to ordinary PF scheduler with different CQI reporting schemes and different power coefficients (M1-M7) for the proposed scheduler. Dual-antenna MRC UE receiver case.

\begin{tabular}{|c|c|c|c|c|c|c|}
\hline & \multicolumn{3}{|c|}{ Coverage Gain [\%] } & \multicolumn{3}{|c|}{ Throughput Loss [\%] } \\
\hline & full & best-m & threshold & full & best-m & threshold \\
\hline M1 & 54 & 57 & 63 & 16 & 16 & 19 \\
\hline M2 & 40 & 42 & 51 & 10 & 10 & 12 \\
\hline M3 & 23 & 26 & 33 & 6 & 6 & 7 \\
\hline M4 & 16 & 18 & 25 & 3 & 4 & 5 \\
\hline M5 & 11 & 14 & 11 & 2 & 3 & 3 \\
\hline M6 & 6 & 7 & 8 & 1 & 2 & 2 \\
\hline M7 & -2 & 0 & -4 & 0 & 0 & 0 \\
\hline
\end{tabular}

Table 4. Obtained performance statistics compared to ordinary PF scheduler with different CQI reporting schemes and different power coefficients (M1-M7) for the proposed scheduler. Dual-antenna IRC UE receiver case.

\begin{tabular}{|c|c|c|c|c|c|c|}
\hline & \multicolumn{3}{|c|}{ Coverage Gain [\%] } & \multicolumn{3}{|c|}{ Throughput Loss [\%] } \\
\hline & full & best-m & threshold & full & best-m & threshold \\
\hline M1 & 56 & 58 & 64 & 15 & 15 & 18 \\
\hline M2 & 43 & 46 & 48 & 9 & 9 & 11 \\
\hline M3 & 26 & 30 & 32 & 6 & 6 & 8 \\
\hline M4 & 17 & 20 & 24 & 4 & 4 & 5 \\
\hline M5 & 10 & 12 & 13 & 2 & 3 & 3 \\
\hline M6 & 8 & 10 & 8 & 2 & 2 & 2 \\
\hline M7 & -1 & 1 & 1 & 0 & 1 & 0 \\
\hline
\end{tabular}




\subsection{Dual Antenna IRC UE Receiver Case}

Next similar performance statistics are obtained for dual antenna interference rejection combining (IRC) UE receiver case. Starting from the primary case M1, with full CQI, we obtain a $13 \%$ loss in throughput and $57 \%$ coverage improvement. For the reduced feedback reporting schemes - best-m and threshold based - we have $13 \%$ and $15 \%$ throughput losses and $58 \%$ and $62 \%$ coverage gains, respectively. Furthermore, resource allocation gains for full CQI feedback and best-m are 7\% for M1 and $17 \%$ for M2 correspondingly. The average obtained improvement for the rest of the cases is about $34 \%$. Threshold based reporting scheme leads to decrease of $12 \%$ for $\mathrm{M} 1$ and $7 \%$ for M2, and roughly $14 \%$ increase for the rest of simulated cases. The exact percentage
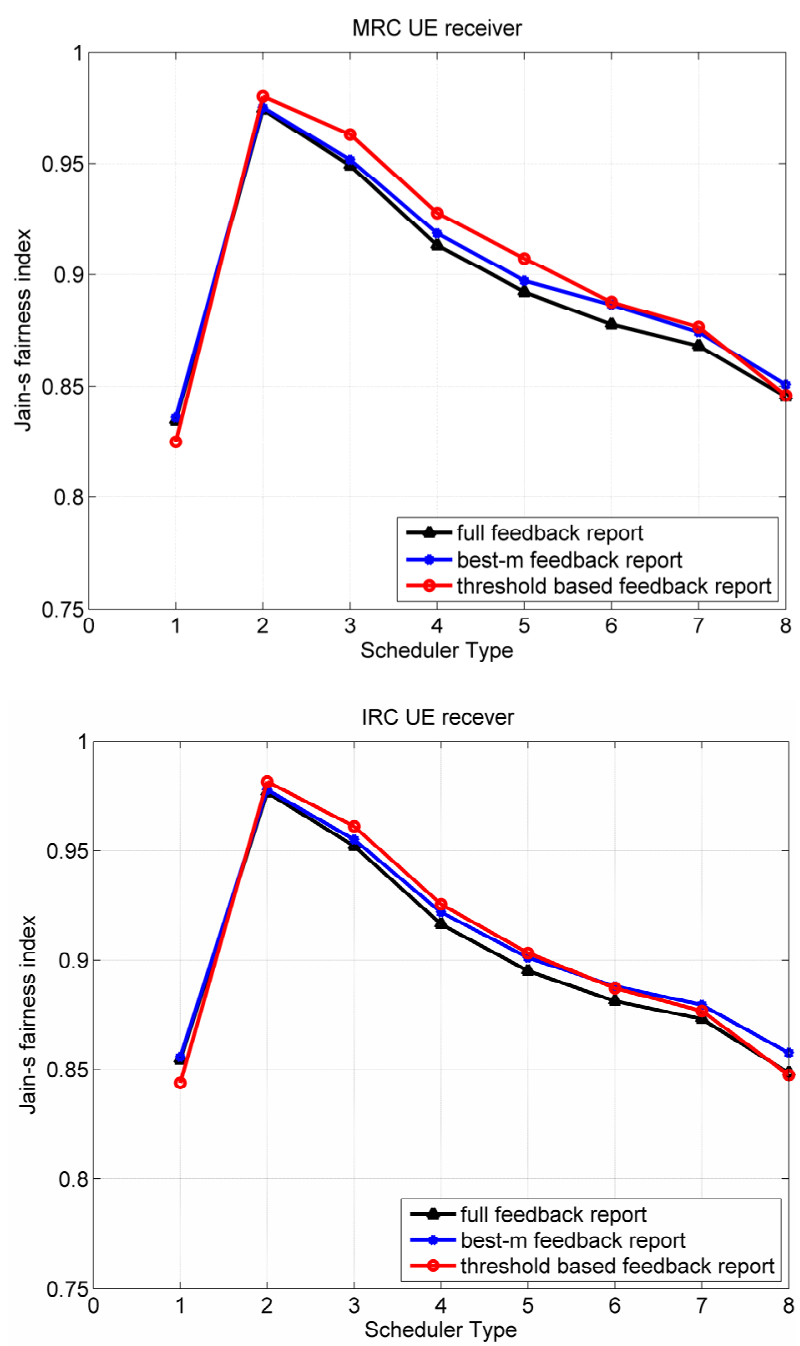

Figure 6. Jain's fairness index per feedback reporting scheme for dual-antenna MRC UE receiver case (up) and dual-antenna IRC UE receiver case (down). Scheduler type 1 means ordinary PF, while 2-8 means proposed modified PF with power coefficients as described in Table 2. read from the figures are again stated in table format in Table 4 in the end.

\subsection{Fairness Index}

Figure 6 illustrates the Jain's fairness index per scheduler for the applied feedback reporting schemes, calculated over all the $I_{\mathrm{TOT}}=20$ UE's using the truly realized UE throughputs at each TTI and over all the simulation runs. The value on the $\mathrm{x}$-axis corresponds to the used scheduler type, where 1 refers to the reference PF scheduler and 2-8 refer to the proposed modified PF schedulers with different power coefficients. The Jain's fairness index defined in [19] is generally in the range of [0...1], where the value of 1 corresponds to all users having the same amount of resources (maximum fairness). Clearly, the fairness distribution with the proposed modified PF scheduler outperforms the used reference PF scheduler for both UE receiver types. The received fairness gains are in range of $2 \%-17 \%$ for the MRC receiver case, and $1 \%-14 \%$ for the IRC receiver case, respectively.

\section{Conclusions}

In this article, we have studied the potential of advanced packet scheduling principles in OFDMA type radio system context, using UTRAN long term evolution (LTE) as a practical example system scenario. A modified proportional fair scheduler taking both the instantaneous channel qualities (CQI's) as well as resource allocation fairness into account was proposed. Also different practical CQI reporting schemes were discussed, and used in the system level performance evaluations of the proposed scheduler. All the performance evaluations were carried out with a comprehensive quasi-static system level simulator, conforming fully to the current LTE working assumptions. Also different UE receiver types were demonstrated in the performance assessments. In general, the achieved throughput and coverage gains were assessed against more traditional ordinary proportional fair scheduling. In the case of fixed coverage requirements and based on the optimal parameter choice for CQI reporting schemes, the proposed scheduling metric calculations based on UE channel feedback offers better control over the ratio between the achievable cell/UE throughput and coverage increase. As a practical example, even with limited CQI feedback, the cell coverage can be increased significantly (more than $30 \%$ ) by allowing a small decrease (in the order of only $5-10 \%$ ) in the cell throughput. This is seen to give great flexibility to the overall RRM process and optimization.

\section{Acknowledgments}

Fruitful discussions with Markku Kuusela, Nokia Devices, Helsinki, Finland, and Dr. Toni Huovinen, Tam- 
pere University of Technology, Tampere, Finland, are greatly acknowledged.

\section{References}

[1] 3GPP RAN Technical Specification Group, "E-UTRA/ E-UTRAN Overall description, stage 2," Technical Report TR 36.300, ver. 9.0.0, June 2009.

[2] 3GPP RAN Technical Specification Group, "E-UTRA/ LTE physical layer-General description," Technical Report TR 36.201, ver. 8.3.0, March 2009.

[3] 3GPP RAN Technical Specification Group, "Physical layer aspects for evolved UTRA," Technical Report TR 25.814, ver. 7.1.0, Oct. 2006.

[4] N. D. Tripathi, et al., "Radio resource management in cellular systems," Springer, 2001.

[5] H. Holma and A. Toskala, Eds., "HSDPA/HSUPA for UMTS-High speed radio access for mobile communications,” Wiley, 2006.

[6] E. Dahlman, et al., "3G evolution: HSPA and LTE for mobile broadband," Academic Press, 2007.

[7] S. Yoon, C. Suh, Y. Cho, and D. Park, "Orthogonal frequency division multiple access with an aggregated subchannel structure and statistical channel quality measurements," in Proc. IEEE Vehicular Technology Conference (VTC'04 Fall), Los Angeles, CA, September 2004.

[8] Y. Sun, et al., "Multi-user scheduling for OFDMA downlink with limited feedback for evolved UTRA," in Proc. IEEE Vehicular Technology Conference (VTC'06 Fall), Montreal, Canada, September 2006.

[9] I. Toufik and H. Kim, "MIMO-OFDMA opportunistic beamforming with partial channel state information," in Proc. IEEE International Conference on Communications, Instanbul, Turkey, pp. 5389-5394, June 2006.

[10] T. E. Kolding, F. Frederiksen, and A. Pokhariyal, "Lowbandwidth channel quality indication for OFDMA frequency domain packet scheduling," in Proc. ISWCS'06, Spain, September 2006.

[11] K. I. Pedersen, G. Monghal, I. Z. Kovacs, T. E. Kolding, A. Pokhariyal, F. Frederiksen, and P. Mogensen, "Frequency domain scheduling for OFDMA with limited and noisy channel feedback," in Proc. IEEE Vehicular Technology Conference (VTC'07 Fall), Baltimore, MD, pp. 1792-1796, Sept. 2007.

[12] P. Svedman, D. Hammarwall, and B. Ottersten, "Subcarrier SNR estimation at the transmitter for reduced feedback OFDMA," in Proc. European Signal Processing Conf., Florence, Italy, September 2006.
[13] C. Wengerter, J. Ohlhorst, and A. G. E Von Elbwert," Fairness and throughput analysis for generalized proportional fair frequency scheduling in OFDMA," in Proc. IEEE Vehicular Technology Conference (VTC' 05 Spring), Stockholm, Sweden, May 2005.

[14] S. Nonchev, J. Venäläinen, and M. Valkama, "New frequency domain packet scheduling schemes for UTRAN LTE Downlink," in Proc. ICT Mobile Summit, Stockholm, Sweden, June 2008.

[15] S. Nonchev and M. Valkama, "Efficient packet scheduling schemes for multiantenna packet radio downlink," in Proc. Fifth Advanced Int. Conf. Telecommunications (AICT’09), Venice, Italy, May 2009.

[16] T. E. Kolding, "Link and system performance aspects of proportional fair scheduling in WCDMA/HSDPA," in Proc. IEEE Vehicular Technology Conference (VTC'03 Fall), Orlando, FL, pp. 1717-1723, Oct. 2003.

[17] A. Pokhariyal, K. I. Pedersen, G. Monghal, I. Z. Kovacs, C. Rosa, T. E. Kolding, and P. E. Mogensen, "HARQ aware frequency domain packet scheduler with different degrees of fairness for the UTRAN long term evolution," in Proc. IEEE Vehicular Technology Conference (VTC' 07 Spring), Dublin, Ireland, April 2007, pp. 2761-2765.

[18] A. Pokhariyal, T. E. Kolding, and P. E. Mogensen, "Performance of downlink frequency domain packet scheduling for the UTRAN long term evolution," in Proc. IEEE Personal, Indoor and Mobile Radio Communications Conference (PIMRC’06), Helsinki, Finland, Sept. 2006.

[19] D. Chui and R. Jain, "Analysis of the increase and decrease algorithms for congestion avoidance in computer networks," Computer Networks and ISDN Systems, 1989.

[20] P. Svedman, S. K. Wilson, L. J. Cimini, and B. Ottersten, "A simplified opportunistic feedback and scheduling scheme for OFDMA," in Proc. IEEE Vehicular Technology Conference (VTC'04 Spring), pp. 1878-1882, May 2004.

[21] P. Svedman, L. J. Cimini, and B. Ottersten, "Using unclaimed sub-carriers in opportunistic OFDMA systems," in Proc. IEEE Vehicular Technology Conference (VTC' 06 Fall), Montreal, Canada, September 2006.

[22] S. Sanayei, A. Nosratinia, and N. Aldhahir, "Opportunistic dynamic sub-channel allocation in multiuser OFDM networks with limited feedback," in IEEE Proc. Inform. Theory Workshop, San Antonio, TX, pp. 182-186, October 2004.

[23] P. Morgensen, et al., "LTE capacity compared to the Shannon bound," in Proc. IEEE Vehicular Technology Conference (VTC'07 Spring), Dublin, Ireland, April 2007. 